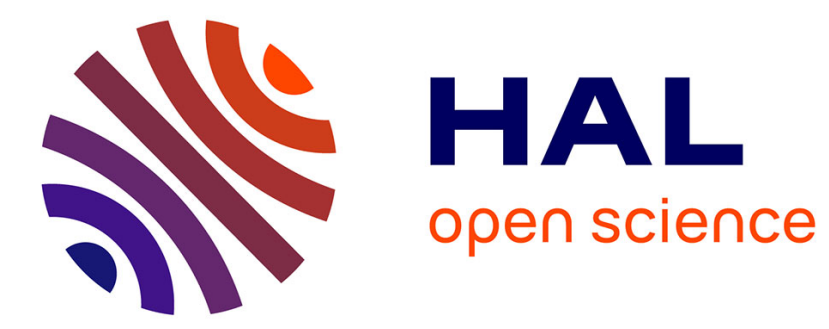

\title{
STEREOCHEMICAL FEATURES OF THE PHYSISORPTION OF LINEAR MOLECULES IN $\beta$-CYCLODEXTRIN
}

Elena Alvira

\section{- To cite this version:}

Elena Alvira. STEREOCHEMICAL FEATURES OF THE PHYSISORPTION OF LINEAR MOLECULES IN $\beta$-CYCLODEXTRIN. Supramolecular Chemistry, 2011, pp.1. 10.1080/10610278.2011.566615 . hal-00693829

\section{HAL Id: hal-00693829 \\ https://hal.science/hal-00693829}

Submitted on 3 May 2012

HAL is a multi-disciplinary open access archive for the deposit and dissemination of scientific research documents, whether they are published or not. The documents may come from teaching and research institutions in France or abroad, or from public or private research centers.
L'archive ouverte pluridisciplinaire HAL, est destinée au dépôt et à la diffusion de documents scientifiques de niveau recherche, publiés ou non, émanant des établissements d'enseignement et de recherche français ou étrangers, des laboratoires publics ou privés. 


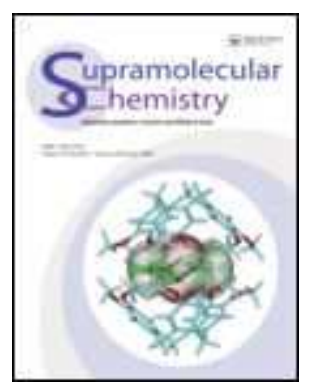

\section{STEREOCHEMICAL FEATURES OF THE PHYSISORPTION OF LINEAR MOLECULES IN $\beta$-CYCLODEXTRIN}

\begin{tabular}{|r|l|}
\hline Journal: & Supramolecular Chemistry \\
\hline Manuscript ID: & GSCH-2010-0229.R1 \\
\hline Manuscript Type: & Full Paper \\
\hline Date Submitted by the \\
Author: & $16-$ Feb-2011 \\
\hline Complete List of Authors: & Alvira, Elena; Universidad de La Laguna, Fisica Fundamental II \\
\hline Keywords: & $\begin{array}{l}\text { computational chemistry, molecular modeling, continuum model, } \\
\text { inclusion complex, macromolecule }\end{array}$ \\
\hline
\end{tabular}

\section{SCHOLARONE ${ }^{\text {m }}$ Manuscripts}




\title{
STEREOCHEMICAL FEATURES OF THE PHYSISORPTION OF LINEAR MOLECULES IN $\beta$-CYCLODEXTRIN
}

\begin{abstract}
E. Alvira
The aim of the present work is to analyse the dependence of the interaction energy between $\beta-\mathrm{CD}$ and linear guest molecules on the atomic distribution of the latter. The shape of the interaction potential does not depend on the symmetry of the molecular configuration, but it is related to the position of the larger atoms in the linear guest.
\end{abstract}
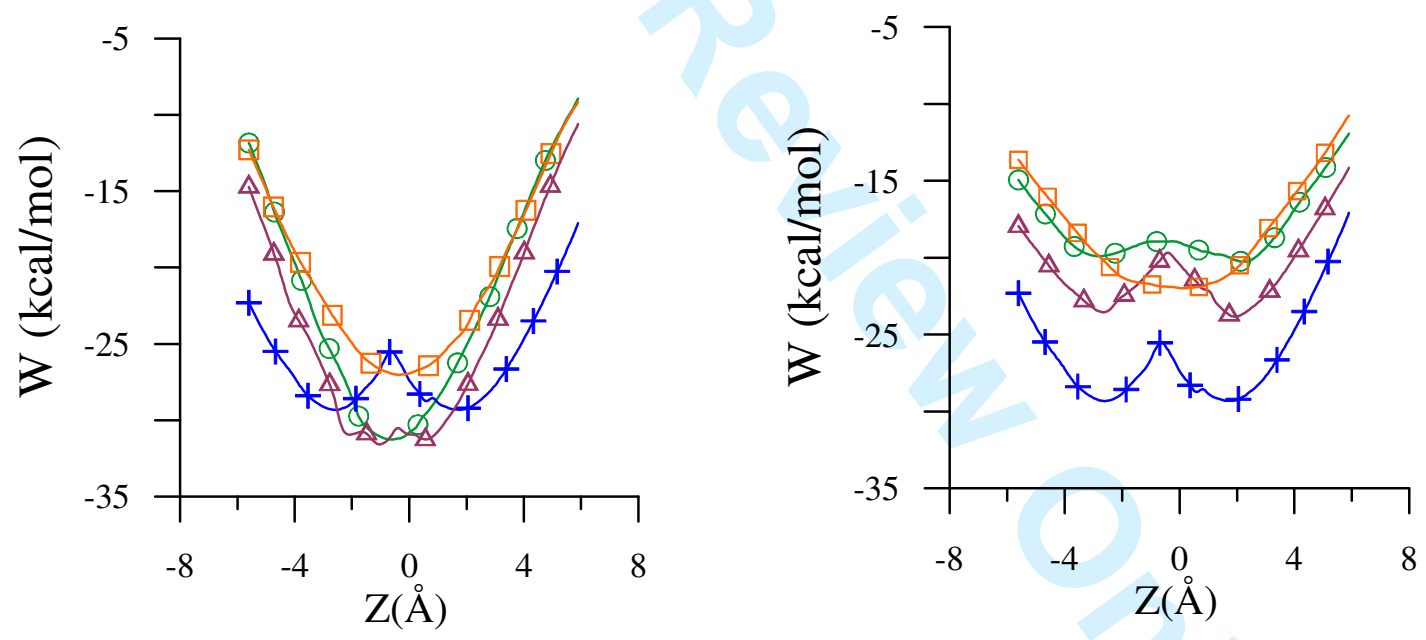


\section{STEREOCHEMICAL FEATURES OF THE}

\section{PHYSISORPTION OF LINEAR MOLECULES IN $\beta$ -}

\section{CYCLODEXTRIN}

\section{E. Alvira}

Departamento de Física Fundamental II

Universidad de La Laguna

38206 La Laguna. Tenerife. Spain

malvira@ull.es 
Abstract

The aim of the present work is to analyse the dependence of the interaction energy between $\beta$-cyclodextrin and linear guest molecules on the atomic distribution of the latter. The intermolecular energy is modelled by a Lennard-Jones potential where the molecular composition is represented by two different pairs of parameters $\left(\sigma_{1}, \varepsilon_{1} ; \sigma_{2}, \varepsilon_{2}\right)$, and a continuum description of the guest and cavity walls. The shape of the interaction potential does not depend on the symmetry of the molecular configuration, but it is related to the position of the larger atoms in the linear guest. For the differences in the interaction energy between isomers to be appreciable, the molecule must be longer than $8 \AA$ and there must be a sudden change rather than multiple variations in the atomic size.

Keywords: computational chemistry, molecular modeling, continuum model, inclusion complex, macromolecule. 


\section{Introduction}

Cyclodextrins (CDs) are macrocyclic molecules composed of glucose units (7 in $\beta$-CD) forming truncated cone-shaped compounds. Their capacity for catalysis and chiral recognition is due mainly to the formation of inclusion complexes, when some lipophilic part of a molecule enters the hydrophobic cyclodextrin cavity [1, 2]. The type of molecules that can be introduced in the cavity to form an inclusion complex, depends mainly on geometric factors rather than on chemical properties, however these are evidently not the only factors, it is shown that the ability of CDs to form supramolecular assemblies is also due to the effect of conformational adaptation of the host and the guest molecules. In some cases the CD hardly modifies his atomic positions by the inclusion complex formation $[3,4]$, but there are some compounds capable of changing the truncated cone shaped structure of CDs to nanocylinder-like blocks $[5,6]$. The present work considers the $\mathrm{CD}$ cavity like a conical geometry, to take into account these structural changes it could be applied the characteristics of a continuum model for the interaction energy of a cylindrical structure for CDs studied in previous work [7].

In the last few years there has been an increase in bionanotechnology research involving CDs, given that their ability to discriminate between enantiomers like ibuprofen [8] makes them particularly useful as molecular adapters in stochastic biosensors. Therefore, characterising the stereochemistry of their interaction with potential analyte molecules is important. Although CDs are often employed in chiral separations [2-11], they are equally applicable to separations involving other molecular geometry differences such as structural and positional isomers [12-14]. However, these

Deleted:

Deleted: 3

Deleted: 5

Deleted: 6

Deleted: 8

studies focus on particular systems and there is no comparative study of the influence of 
atomic distribution on the interaction energy between guest and host, to determine the decisive factors giving rise to different inclusion complex configurations for the isomers.

The van der Waals term is the main contribution to the total energy, particularly inside the cavity, so it directly determines the configuration of the inclusion complex [15-17]. This potential energy represents the attractive and repulsive interactions acting between all atoms and molecules, even totally neutral ones; in contrast to other types of forces present, or not, according to the properties of the molecules, such as electrostatic charges, dipoles or hydrogen bonds. To take into account the effects of the solvent, other interaction energies must be considered. An example of the way in which the entropy of the solvent affects the interaction between solute molecules is the hydrophobic effect, which is one of the driving forces responsible of the inclusion complex formation with CDs. On the theoretical side, the hydrophobic interaction between two molecules is much more complex because it is of longer range than that arising from any simple additive potential, and the hydrogen bonds network must be included in any computer simulation $[18,19]$.

In previous work, we proposed a model for the interaction energy between $\beta-\mathrm{CD}$ and guest molecules of different structure and size [20-22]. The intermolecular energy was then modelled by a Lennard-Jones potential and a continuum description of the guest and cavity walls. This simple model is able to reproduce several quantitative and qualitative features of the interaction energy between $\beta-\mathrm{CD}$ and cyclic, spherical or linear molecules. To simulate the potential energy for linear guests obtained by standard methods of molecular mechanics, in that study we proposed two possible ways to represent the composition of the guest: one pair of potential parameters $(\sigma, \varepsilon)$ [20], 
where the parameter $\varepsilon$ governs the strength of the interaction and $\sigma$ defines a length scale, or two pairs $\left(\sigma_{1}, \varepsilon_{1} ; \sigma_{2}, \varepsilon_{2}\right)$ [21]. The penetration potential is different in both cases, a well potential was found for the former, whereas the latter presented the two minima inside the cavity, separated by a potential barrier deriving from a rotation of the linear molecule. In the model of guest molecule considered in that work, the atoms whose interaction with $\beta$-CD is characterized by $\left(\sigma_{1}, \varepsilon_{1}\right)$ were distributed together, from the top to the centre of the linear molecule. To take into account a different distribution of atoms along the guest molecule, a new parameter is necessary to study the stereochemical features of inclusion complexes formed with linear molecules and $\beta-C D$, since we can represent some guest molecules with the same composition but different atomic distribution. The resulting configurations give rise to structural isomers not stereoisomers, as a consequence of the geometry and continuum model for the guest.

The shape of the interaction energy for linear molecules is important because the possibility of confinement inside the cavity is related above all to the potential barrier, as we established from the dynamic study of these systems [23]. The different form of interaction energy was assigned to the asymmetric distribution of atoms in the guest, but we did not study the influence of atomic distribution along the linear molecule on the physisorption in $\beta-C D$ using the continuum model.

The aim of the present work is to analyse the dependence of the interaction energy between $\beta-\mathrm{CD}$ and linear molecules on the atomic distribution of the guest. Section 2 presents the model, then the main results and comparison with the all-atom model are discussed in Section 3. The influence of molecular stereochemistry on the mobility of the guest into the cavity will be the aim of a forthcoming work based on molecular dynamics. 


\section{The model}

The intermolecular energy is modelled by a Lennard-Jones potential combined with a continuum description of the cyclodextrin cavity and the guest molecule. Since continuity is assumed for the guest and the host, this interaction energy is not capable of reproducing the hydrogen bonds formed between the linear molecule and CD. In the present study, we consider the interaction between the atoms of the system composed of two different parameter pairs $\left(\sigma_{1}, \varepsilon_{1} ; \sigma_{2}, \varepsilon_{2}\right)$ and linear molecules with length $L \geq 5 \AA$. The molecular composition is characterized by these potential parameters and the ratio $p$ of each pair, $0 \leq p \leq 1, p=0$ being when the interaction is represented by the values $\left(\sigma_{2}, \varepsilon_{2}\right)$ and $p=1$ corresponds to the pair $\left(\sigma_{1}, \varepsilon_{1}\right)$. In the model of guest molecule considered in previous work, the atoms whose interaction with $\beta-\mathrm{CD}$ is characterized by $\left(\sigma_{1}, \varepsilon_{1}\right)$ are distributed together, from the top to the centre of the linear molecule [21]. To consider a different distribution of atoms along the guest molecule, for instance $\left(\sigma_{1}, \varepsilon_{1}\right),\left(\sigma_{2}, \varepsilon_{2}\right),\left(\sigma_{1}, \varepsilon_{1}\right)$, new parameters $m_{i}$ are necessary to represent the positions of different compositions on the linear molecule. Therefore the interaction energy $W$ can be calculated as:

$$
\begin{aligned}
& \qquad W\left(\vec{r}_{m o l}\right)=\rho_{C D} \rho_{m o l}\left[\int_{-\frac{L}{2}}^{m_{1}} d \vec{l} \int_{S} V_{1}\left(\vec{r}_{l}, \vec{r}_{C D}\right) d \vec{r}+\int_{m_{1}}^{m_{2}} d \vec{l} \int_{S} V_{2}\left(\vec{r}_{l}, \vec{r}_{C D}\right) d \vec{r}+\right. \\
& \left.+\int_{m_{2}}^{\frac{L}{2}} d \vec{l} \int_{S} V_{1}\left(\vec{r}_{l}, \vec{r}_{C D}\right) d \vec{r}\right] \\
& \text { with }
\end{aligned}
$$


where the guest molecule-CD interaction is represented by an average of the uniformly distributed atoms in the $\mathrm{CD}$ and in the linear molecule. $\rho_{C D}$ is the superficial density of atoms in the CD cavity, $\rho_{m o l}$ is the linear density of atoms in the guest molecule, $d \vec{l}$ is the differential of length (on the linear molecule) located at $\vec{r}_{l}$, and $d \vec{r}$ is the differential of surface (on the cavity) located at $\vec{r}_{C D}$ (Fig. 1). The interaction energy integral $W\left(\vec{r}_{m o l}\right)$ cannot be obtained as an analytical expression, it is necessary to resolve it by numerical methods even if the guest is located along the cavity axis. $W\left(\vec{r}_{\text {mol }}\right)$ depends on the length $L$ of the linear molecule, its composition and the distribution of atoms in it. In previous work we have analysed the influence of molecular length $L, p$ and the parameters $\left(\sigma_{1}, \varepsilon_{1} ; \sigma_{2}, \varepsilon_{2}\right)$ on the interaction energy; the present study is focused on the influence of atomic distribution on $W\left(\vec{r}_{\text {mol }}\right)$.

The linear molecule can be symmetric or asymmetric, depending on the atomic distribution, for instance when $m_{1}=-m_{2}$ and $m_{2}=(1-p) \frac{L}{2}$ the atoms whose interaction with $\beta-\mathrm{CD}$ is characterized by $\left(\sigma_{2}, \varepsilon_{2}\right)$ are distributed together around the centre of the linear molecule, which turns out to be a symmetric molecule.

We place the origin of the reference system at the centre of mass of the $\mathrm{CD}$ and the space-fixed frame over the principal axis of the $\beta-\mathrm{CD}$. The configuration of the linear guest $\vec{r}_{m o l}$ is given by the coordinates of its centre of mass $\vec{r}_{0}$ and its molecular orientation. The latter is defined by the polar angles $(\theta, \varphi)$ formed with respect to the 
absolute frame $(X, Y, Z)$. For all the atoms, the interaction energy is determined at each position of the centre of mass $\vec{r}_{0}$ after minimization over the Euler angles of the guest molecule [24]. In the present model, the potential energy is also calculated for different molecular orientations which result in rotation of the two polar angles at intervals of $6^{\circ}$ and $12^{\circ}$ respectively (about 900 orientations), and the minimum for these values is assigned to the position $\vec{r}_{0}=\left(x_{0}, y_{0}, z_{0}\right)$. Since we are dealing with a conical continuum geometry, for every plane $Z=$ constant, the potential energy is the same for points $\left(x_{0}, y_{0}\right)$ located at the same distance $d$ from the cavity axis $\left(x_{0}^{2}+y_{0}^{2}=d^{2}\right)$. In these positions, the linear molecule adopts different orientations to minimize the energy: while the polar angle formed with the cavity axis $\theta$ is the same, the value of $\varphi$ is different from one point to another. Therefore, some configurations of the guest molecule $\left(\vec{r}_{0}, \theta, \varphi\right)$ are equivalent in that they have the same energy; for this reason we characterize the guest configuration for each energy by the centre of mass position (defined by the distance $d$ from the cavity axis and the $Z$ coordinate) and the molecular orientation (characterized only by the polar angle $\theta$ ).

The potential energy is determined by Eq. (1) for different configurations of the guest $\vec{r}_{\text {mol }}$, inside and outside the CD. In each plane $Z=$ constant, about 500 points are explored (the distance between two consecutive points for each axis being $0.2 \AA$ ), and the range of variation along the $Z$ axis is about $10 \AA$ (with a path equal to $0.1 \AA$ ). The results obtained are represented by the potential energy surfaces, penetration potentials and the inclusion complex configuration, as in previous work [20-22]. The curve joining the minimum potential energy for every plane $Z=$ constant defines the penetration potential, which describes the variation in interaction energy when its path through the
Deleted: 16

Deleted: defined by the two polar angles 
cavity is non-axial. The position of the guest molecule for which we obtain the absolute minimum potential energy gives the geometry of the inclusion complex.

The potential energy surfaces are represented by partitioning the variation range of the $Z$ axis in the $\beta$-CD cavity into four parts, depending on the position of the guest molecule centre of mass: near the top of the cone, near the centre of the cavity and near the cone base. The length of each domain is about $2 \AA$ and the potential surface for each region is determined as the minimum energy for every point on the plane in the corresponding interval of $Z$.

\section{Results and discussion}

The present study performs a comparative study of the function $W\left(\vec{r}_{m o l}\right)$ for guest molecules with different atomic distributions and configurations (orientation and centre of mass position represented by $\left.\vec{r}_{m o l}\right)$. We consider 7 cases $\left(\sigma_{1}\right.$ being $\left.<\sigma_{2}\right)$ : $(a)$ the atoms whose interaction with $\beta-\mathrm{CD}$ is characterized by $\left(\sigma_{1}, \varepsilon_{1}\right)$ are distributed together, from the top to the centre of the linear molecule; $(b)$ an asymmetric atomic distribution $\left(\sigma_{1}, \varepsilon_{1}\right),\left(\sigma_{2}, \varepsilon_{2}\right),\left(\sigma_{1}, \varepsilon_{1}\right)$, with $\left(\sigma_{2}, \varepsilon_{2}\right)$ at $\frac{L}{4} ;(c)$ a symmetric atomic distribution $\left(\sigma_{1}, \varepsilon_{1}\right),\left(\sigma_{2}, \varepsilon_{2}\right),\left(\sigma_{1}, \varepsilon_{1}\right)$, with $\left(\sigma_{2}, \varepsilon_{2}\right)$ around the centre of the guest; $(d)$ a symmetric atomic distribution $\left(\sigma_{1}, \varepsilon_{1}\right),\left(\sigma_{2}, \varepsilon_{2}\right),\left(\sigma_{1}, \varepsilon_{1}\right),\left(\sigma_{2}, \varepsilon_{2}\right),\left(\sigma_{1}, \varepsilon_{1}\right) ;(e)$ same as $b$ with the composition $\left(\sigma_{2}, \varepsilon_{2}\right),\left(\sigma_{1}, \varepsilon_{1}\right),\left(\sigma_{2}, \varepsilon_{2}\right) ;(f)$ the same as $c$ with symmetric atomic distribution $\left(\sigma_{2}, \varepsilon_{2}\right),\left(\sigma_{1}, \varepsilon_{1}\right),\left(\sigma_{2}, \varepsilon_{2}\right) ;(g)$ a symmetric atomic distribution 
$\left(\sigma_{2}, \varepsilon_{2}\right),\left(\sigma_{1}, \varepsilon_{1}\right),\left(\sigma_{2}, \varepsilon_{2}\right),\left(\sigma_{1}, \varepsilon_{1}\right),\left(\sigma_{2}, \varepsilon_{2}\right)$. Some samples of real molecules are

\section{represented in Figure 2.}

Figures $3 \mathrm{a}$ and $4 \mathrm{a}$ show the variation in the potential energy along the cavity axis

for a linear molecule with $L=10 \AA, \sigma_{1}=2.5 \AA, \varepsilon_{1}=0.07 \mathrm{kcal} / \mathrm{mol}, \sigma_{2}=3.5 \AA$, $\varepsilon_{2}=0.09 \mathrm{kcal} / \mathrm{mol}, p=0.6$ and different atomic distributions. The values of energy minima and guest configurations $(d, z, \theta)$ in the inclusion complexes are included in Table 1. One type of curve looking like a well potential can be seen and another which presents two minima separated by a potential barrier. There are symmetric atomic distributions like $f$ whose interaction energy with $\mathrm{CD}$ presents two minima and asymmetric molecules like $b$ whose energy resembles a well potential, therefore the shape of the interaction energy is not a consequence of the symmetry of the atomic distribution in the guest.

There are some common features in the interaction energy for guests with the smaller atoms at the top of the linear molecule ( $b$ and $c$ ) and different from the distributions $e$ and $f$; the cases $d$ and $g$ also have similar behaviour but different in turn from the rest. In previous work we verified that the potential barrier for an asymmetric molecule is due to a rotation of the guest of about $180^{\circ}$ with respect to the cavity axis [21]. The molecules for which the intermolecular energy looks like a well potential (b and $c$ ) also present configurations for $Z \leq 0$ rotated about $180^{\circ}$ with respect to those for $Z>0$, and since these cases correspond to distributions where the smaller atoms are at the top of the guest there are small differences in the intermolecular energy between the two types of configuration. Therefore the difference in the shape of the interaction potential does not depend on the symmetry of the atomic distribution, but rather on the position of the larger atoms in the linear guest. 
The least difference occurs between the cases $b$ and $c$, which also contain the absolute minimum. The deeper intermolecular energy for these distributions is a consequence of the guest configuration in the inclusion complex, where the linear molecule is located near the cavity centre, forming an angle of about $30^{\circ}$ with the cavity axis (Table1), while molecules $e$ and $f$ are located near the wide rim of the CD nearly parallel to the cavity axis and therefore further from the cavity walls.

The $Z$ coordinate of the potential barrier is nearly the same for $e$ and $f$ (also for a) (Fig. 4a), but the height of these barriers decreases as the symmetry of atomic distribution increases. On the contrary, the $Z$ coordinate of the relative minima for these cases tends toward positions nearer the $\mathrm{CD}$ rims as the potential barrier decreases.

The molecules $d$ and $g$ show unusual behaviour because the shape of the penetration potential does not depend on the position of the largest atoms on the guest, as in the other cases. We can conclude that the energy reflects a sudden change better than multiple variations in the atomic size. There are no great differences in the inclusion complex formed with the molecules $d$ and $g$, although the differences in angle $\theta$ of guest configurations allow them to approach the $\mathrm{CD}$ and lower the interaction energy for $d$.

Figures $3 \mathrm{~b}$ and $4 \mathrm{~b}$ show the variation in the potential energy along the cavity

axis for a linear molecule with $L=7 \AA, \sigma_{1}=2.5 \AA, \varepsilon_{1}=0.07 \mathrm{kcal} / \mathrm{mol}, \sigma_{2}=3.5 \AA$, $\varepsilon_{2}=0.09 \mathrm{kcal} / \mathrm{mol}, p=0.6$ and different atomic distributions. In this case only the curve for the molecule $a$ really shows a different shape from the rest, because the molecular length is insufficient to distinguish the atomic distributions. The relation between the minimum energies of the different distributions is the same as for the 
molecule with $L=10 \AA$, although the magnitude of these differences is smaller, due mainly to the similar guest configuration in the absolute minimum (Table 1).

Figures $3 \mathrm{c}$ and $4 \mathrm{c}$ show the variation in the potential energy along the cavity axis

for a linear molecule with $L=10 \AA, \sigma_{1}=2.5 \AA, \varepsilon_{1}=0.07 \mathrm{kcal} / \mathrm{mol}, \sigma_{2}=3.7 \AA$, $\varepsilon_{2}=0.18 \mathrm{kcal} / \mathrm{mol}, p=0.5$ and different atomic distributions. The interaction energy is lower in this case because the guest is composed of larger atoms, although it can be seen that a 30\% decrease in molecule length leads to less than a 30\% variation in interaction energy, whereas a small variation in composition nearly triples the energy (Table 1). Moreover the differences in the intermolecular potential between the isomers, and the characteristics of the potential barriers or relative minima in $a, e$ and $f$ are similar to those of molecules with the same length and different composition. Therefore we can conclude that the magnitude of the interaction energy depends mainly on the molecular composition rather than the length, although the shape of this curve is a consequence of the atomic distribution. This conclusion is important in order to analyse the influence of atomic distribution on the mobility of linear molecules inside the $\mathrm{CD}$ (in a forthcoming study), because the possibility of confinement or temporary residence inside the cavity depends on the potential barrier, as occurs for the asymmetric linear molecules studied in Ref. 21. The main differences in the guest configuration in the inclusion complexes formed with molecules of the same length and different composition correspond to $b$ : the molecule with $\sigma_{2}=3.5 \AA$ is oriented such that the part with larger atoms is located near the narrow opening of the CD cavity. However this part is near the wide rim in the inclusion complex formed with the guest where $\sigma_{2}=3.7 \AA$, therefore there is a rotation of about $180^{\circ}$ between them. 
To analyse the variations in the interaction energy inside the cavity, not only along the axis, we represent the differences between the potential surfaces near the top of the cone (Fig. 5a), near the centre of the cavity (Figs. 5b, 5c) and near the cone base (Fig. 5d) $[20,21]$ for molecules with $L=10 \AA, \sigma_{1}=2.5 \AA, \varepsilon_{1}=0.07 \mathrm{kcal} / \mathrm{mol}$, $\sigma_{2}=3.5 \AA, \quad \varepsilon_{2}=0.09 \mathrm{kcal} / \mathrm{mol}, p=0.6$ and atomic distributions $c$ and $f$. The \begin{tabular}{|l|} 
Deleted: $3 \mathrm{a}$ \\
Deleted: $3 \mathrm{~b}$ \\
Deleted: $3 \mathrm{c}$ \\
Deleted: $3 \mathrm{~d}$ \\
Deleted: 12 \\
\hline Deleted: 13
\end{tabular} equipotential zones where the molecule $c$ is more stable than $f$ are represented by solid curves (red) and those where the energy is deeper for $f$ as dashed curves (blue). The greatest differences occur near the cavity walls, whereas near the cavity centre the potential energy is approximately the same. These results can be applied to other compositions, therefore a molecule with smaller atoms located at its top tends to locate its centre of mass nearer the cavity centre forming a greater angle with the cavity axis than a molecule with larger atoms at its extremes.

In previous work we determined the interaction energy between $\beta-\mathrm{CD}$ and some different molecules, in particular the isomers diethyl fumarate and diethyl maleate using a force field method [24]. The results allowed us to determine the potential parameters representing some of these molecules in the continuum model, as with diethyl fumarate [21], but we could not simulate the interaction energy between $\beta-\mathrm{CD}$ and diethyl maleate despite both isomers having the same composition. The results obtained in the present study indicate that the difference in the interaction energy of the isomers is due to the atomic distribution: type $a$ for diethyl fumarate and between $b$ and $d$ for diethyl maleate.

\section{Conclusions}


The present work has analysed how the interaction energy between $\beta-\mathrm{CD}$ and linear molecules depends on the atomic distribution of the guest. The intermolecular energy is modelled by a Lennard-Jones potential where the molecular composition is represented by two different parameter pairs $\left(\sigma_{1}, \varepsilon_{1} ; \sigma_{2}, \varepsilon_{2}\right)$, and a continuum description of the guest and cavity walls. We conclude that the orientation of the guest changes about $180^{\circ}$ with respect to the cavity axis for positions of its centre of mass at $Z<0$ and $Z>0$, giving rise to representations of the intermolecular energy resembling well potentials or two minima separated by a potential barrier. The magnitude of the interaction energy depends mainly on the molecular composition, although the shape of this interaction potential does not depend on the symmetry of the atomic distribution, but on the position of the larger atoms in the linear molecule. For the differences in the interaction energy between isomers to be appreciable, the molecule must be longer than $8 \AA$ and there must be a sudden change rather than multiple variations in the atomic size.

Inclusion complexes formed with linear molecules with smaller atoms at their tops are more stable than those formed with molecules with the larger atoms at their extremes. In these complexes, the first type of guest tends to locate its centre of mass nearer the cavity centre, forming wider angles with the cavity axis than the molecules with the larger atoms at their extremes. These configurations give rise to great differences in potential energy near the cavity walls, whereas near the cavity centre it is approximately the same.

\section{Acknowledgments}


We are grateful to the Ministerio de Ciencia e Innovación (FIS2009-07890) for their generous financial support. 


\section{References}

[1] Szejtli J.; and Osa T., Comprehensive Supramolecular Chemistry, Ed. Pergamon,

Elsevier, Oxford, 1996

[2] Dodziuk H., Cyclodextrins and their complexes. Chemistry, analytical methods,

applications, Ed. Wiley-VCH, Weinheim, 2006

[3] Liu Y.; Yang Z.-X.; Chen Y. J. Org. Chem. 2008, 73, 5298-5304

[4] Wang Y.; Chung T. S.; Wang H.; Goh S. H. Chem. Ing. Sci. 2009, 64, 5198-5209

[5] Brocos P.; Díaz-Vergara N.; Banquy X.; Pérez-Casas S.; Costas M.; Piñeiro A. J.

\section{Phys. Chem. B 2010, 114, 12455-12467}

[6] Hernández-Pascacio J.; Garza C.; Banquy X.; Díaz-Vergara N.; Amigo A.; Ramos

\section{S.; Castillo R.; Costas M.; Piñeiro A. J. Phys. Chem. B Lett. 2007,111, 12625-12630}

\section{[7] Alvira E. Chem. Phys. Lett. 1997, 267, 221-228}

[8] Kang X.; Cheley S.; Guan X.; Bayley H.; J. Am. Chem. Soc. 2006, 128, 1068410685

[9] Franchi P.; Lucarini M.; Mezzina E.; Pedulli G. F. J. Am. Chem. Soc. 2004, 126, $4343-4354$

[10] Malta L. F. B.; Cordeiro Y.; Tinoco L. W.; Campos C. C.; Medeiros M. E.; Antunes O. A. C. Tetrahedron: Asymmetry 2008, 19, 1182-1188

111]Shishovska M.; Trajkovska V. Chirality 2010, 22, 527-533

[12] Pan J.; Kai G.; Yuan C.; Jin R. Chromatographia 2007, 66, 121-123

[13] B'Hymer C. J. Anal. Chem. 2008, 63, 376-380,

[14] Balomenou I.; Pistolis G. J. Phys. Chem. B 2010, 114, 780-785

[15] Lipkowitz, K. B. J. Chromatogr. A 1995, 694, 15-37

Formatted

Deleted: 3

Deleted: II

Deleted: 7

Deleted: Wang Y.; Chung T. S.; Wang H.; Goh S. H. Chem. Ing. Sci. 2009, 64 5198-5209

Deleted: 8

Deleted: 9

[16] Lipkowitz, K. B. Chem. Rev. 1998, 98, 1829-1873

Deleted: 10 
[17] Lipkowitz, K. B. J. Chromatogr. A 2001, 906, 417-442

[18] Israelachvili J., Intermolecular and Surfaces Forces, Academic Press, San Diego,

1995

[19] Stone A. J., The Theory of Intermolecular Forces, Oxford University Press, New

York, 1996

[20] Alvira, E. Chem. Phys. Lett. 2007, 439, 252-257

[21] Alvira, E. Chem. Phys. Lett. 2007, 439, 258-263

[22] Alvira, E. Supramol. Chem. 2010, 22, 3, 156-162

[23] Alvira, E. Mol. Phys. 2009, 107, 1697-1702

241 Alvira, E. Chem. Phys. Lett. 1997, 271, 178-184
Deleted: 11

Formatted

Formatted

Deleted: 12

Deleted: 13

Deleted: 14

Deleted: 15

Deleted: [16] Alvira, E. Chem. Phys. Lett. 1997, 267, 221-228 


\section{TABLES}

Table 1 . The configuration of the guest and the absolute minimum of energy (in kcal/mol) for the different atomic distributions.

\begin{tabular}{ccccccccccccc}
\hline & \multicolumn{3}{c}{$L=10 \AA, \sigma_{2}=3.5 \AA, p=0.6$} & \multicolumn{3}{c}{$L=7 \AA, \sigma_{2}=3.5 \AA, p=0.6$} & \multicolumn{2}{c}{$L=10 \AA, \sigma_{2}=3.7 \AA ⿻, p=0.5$} \\
& $d(\AA)$ & $\mathrm{Z}(\AA)$ & $\theta$ & $W$ & $d(\AA)$ & $\mathrm{Z}(\AA)$ & $\theta$ & $W$ & $d(\AA)$ & $\mathrm{Z}(\AA)$ & $\theta$ & $W$ \\
\hline$a$ & 1.0 & -2.6 & $24^{\circ}$ & -29.33 & 1.3 & 0.8 & $24^{\circ}$ & -22.93 & 0.2 & -2.6 & $12^{\circ}$ & -78.91 \\
$b$ & 0.2 & -1.1 & $30^{\circ}$ & -31.57 & 0.6 & 0.0 & $36^{\circ}$ & -23.39 & 0.4 & 0.2 & $12^{\circ}$ & -79.64 \\
$c$ & 0.4 & -0.6 & $30^{\circ}$ & -31.27 & 0.4 & -0.4 & $36^{\circ}$ & -23.50 & 0.2 & -0.4 & $12^{\circ}$ & -79.55 \\
$d$ & 0.4 & -0.2 & $18^{\circ}$ & -27.02 & 0.2 & -0.2 & $30^{\circ}$ & -21.37 & 0.4 & 0.0 & $12^{\circ}$ & -70.00 \\
$e$ & 1.6 & 1.9 & $18^{\circ}$ & -23.84 & 0.8 & 0.6 & $12^{\circ}$ & -18.58 & 1.2 & 2.4 & $18^{\circ}$ & -57.40 \\
$f$ & 1.6 & 2.4 & $18^{\circ}$ & -20.23 & 0.6 & -0.2 & $12^{\circ}$ & -17.43 & 1.2 & 2.4 & $18^{\circ}$ & -51.55 \\
$g$ & 0.8 & 0.2 & $12^{\circ}$ & -22.00 & 0.6 & -0.4 & $12^{\circ}$ & -18.57 & 0.4 & -0.4 & $6^{\circ}$ & -58.75 \\
\hline
\end{tabular}




\section{Figure Captions}

Fig. 1 Schematic representation of the coordinates of the $C D$ and linear molecule.

Fig. 2 (a) Diethyl fumarate, molecular structure type $a_{\mathrm{A}}$ (b) Diethyl maleate, between $b$ and $d$, Ref. 24 (c) 1-butanol, type $a$ (d) 2-butanol, type $b$, Ref. 4 (e) 2-[4-(1-hydroxy-4[4-(hydroxy-diphenylmethyl)-1-piperidinyl]-butyl)-phenyl]-2-methylpropionic acid HCL, type a (f) 2-[3-(1-hydroxy-4-[4-(hydroxydiphenylmethyl)-1-piperidinyl]-butyl)phenyll-2-methyl-propionic acid HCL, type between $e$ and f, Ref. 13.

Fig. 3. (a) Variation in the potential energy along the cavity axis for a linear molecule with $L=10 \AA, \sigma_{1}=2.5 \AA, \varepsilon_{1}=0.07 \mathrm{kcal} / \mathrm{mol}, \sigma_{2}=3.5 \AA, \varepsilon_{2}=0.09 \mathrm{kcal} / \mathrm{mol}, p=0.6$ and atomic distributions $a, b, c$ and $d$.

(b) Variation in the potential energy along the cavity axis for a linear molecule with $L=7 \AA, \sigma_{1}=2.5 \AA, \varepsilon_{1}=0.07 \mathrm{kcal} / \mathrm{mol}, \sigma_{2}=3.5 \AA, \varepsilon_{2}=0.09 \mathrm{kcal} / \mathrm{mol}, p=0.6$ and atomic distributions $a, b, c$ and $d$.

(c) Variation in the potential energy along the cavity axis for a linear molecule with $L=10 \AA, \sigma_{1}=2.5 \AA, \varepsilon_{1}=0.07 \mathrm{kcal} / \mathrm{mol}, \sigma_{2}=3.7 \AA, \varepsilon_{2}=0.18 \mathrm{kcal} / \mathrm{mol}, p=0.5$ and atomic distributions $a, b, c$ and $d$.

Fig. 4 (a) Variation in the potential energy along the cavity axis for a linear molecule with $L=10 \AA, \sigma_{1}=2.5 \AA, \varepsilon_{1}=0.07 \mathrm{kcal} / \mathrm{mol}, \sigma_{2}=3.5 \AA, \varepsilon_{2}=0.09 \mathrm{kcal} / \mathrm{mol}, p=0.6$ and atomic distributions $a, e, f$ and $g$.

(b) Variation in the potential energy along the cavity axis for a linear molecule with $L=7 \AA, \sigma_{1}=2.5 \AA, \varepsilon_{1}=0.07 \mathrm{kcal} / \mathrm{mol}, \sigma_{2}=3.5 \AA, \varepsilon_{2}=0.09 \mathrm{kcal} / \mathrm{mol}, p=0.6$ and atomic distributions $a, e, f$ and $g$.

\begin{tabular}{|l|}
\hline Formatted \\
\hline Formatted \\
\hline Formatted \\
\hline Formatted \\
\hline Formatted \\
\hline Formatted \\
\hline Formatted \\
\hline
\end{tabular}

Deleted: 2 
1

2

3

4

5

6

7

8

9

10

11

12

13

14

15

16

17

18

19

20

21

22

23

24

25

26

27

28

29

30

31

32

33

34

35

36

37

38

39

40

41

42

43

44

45

46

47

48

49

50

51

52

53

54

55

56

57

58

59

60

(c) Variation in the potential energy along the cavity axis for a linear molecule with $L=10 \AA, \sigma_{1}=2.5 \AA, \varepsilon_{1}=0.07 \mathrm{kcal} / \mathrm{mol}, \sigma_{2}=3.7 \AA, \varepsilon_{2}=0.18 \mathrm{kcal} / \mathrm{mol}, p=0.5$ and atomic distributions $a, e, f$ and $g$.

Fig. 5 (a) Differences between the potential surfaces near the top of the cone, (b) and (c) near the centre of the cavity and (d) near the cone base for molecules with $L=10 \AA$, $\sigma_{1}=2.5 \AA, \varepsilon_{1}=0.07 \mathrm{kcal} / \mathrm{mol}, \sigma_{2}=3.5 \AA, \varepsilon_{2}=0.09 \mathrm{kcal} / \mathrm{mol}, p=0.6$ and atomic distributions $c$ and $f$. The equipotential zones where the molecule $c$ is more stable than $f$ are represented by solid curves (red) and those where the energy is deeper for $e$ are printed as dashed curves (blue). 


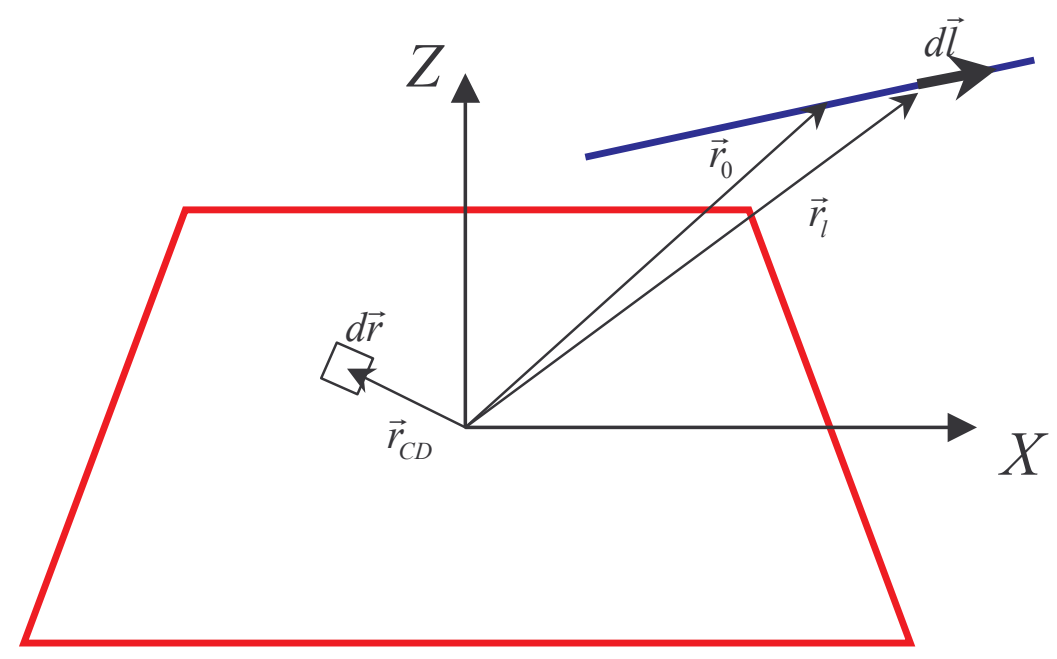

Figure 1 


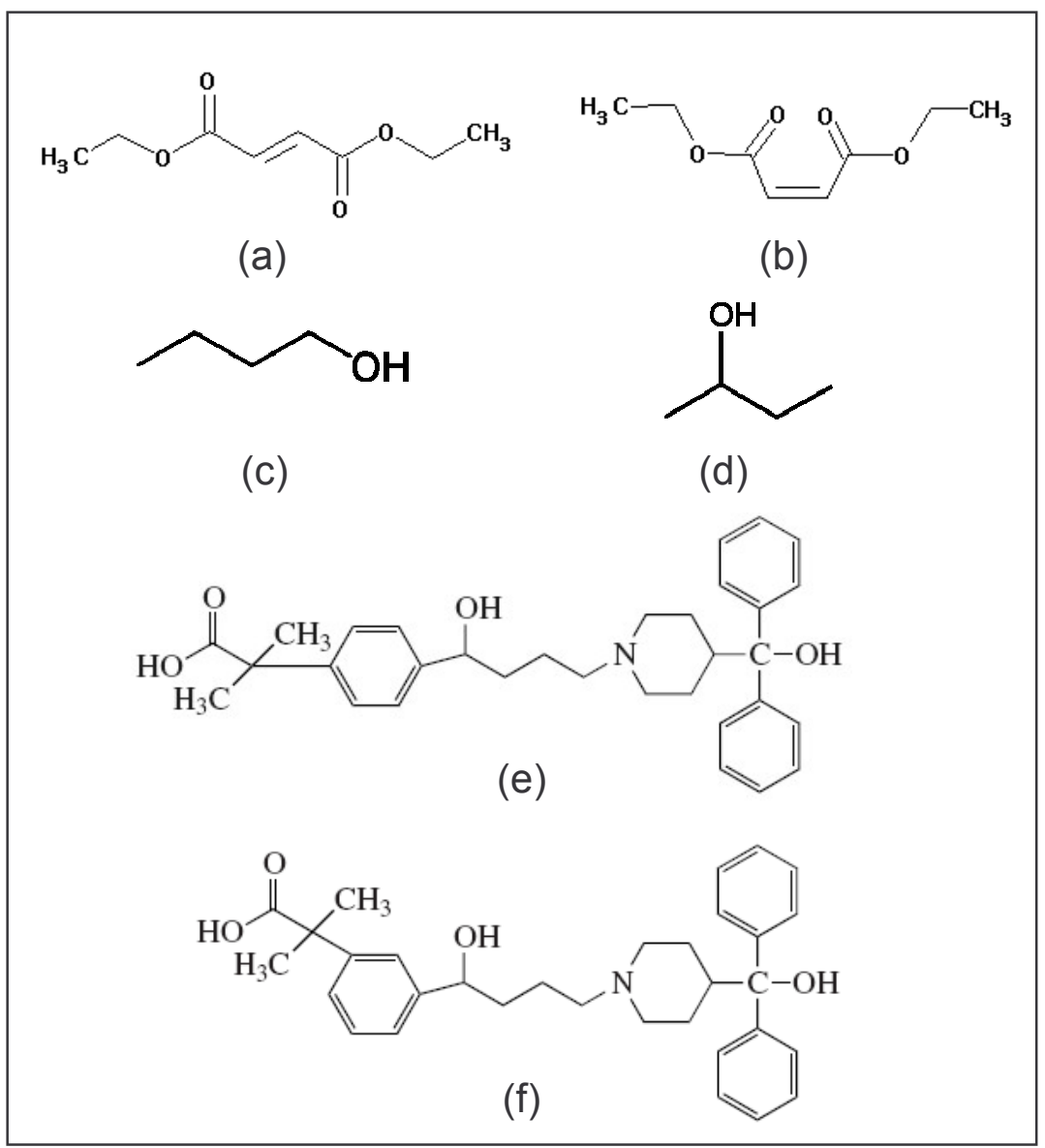

Figure 2 

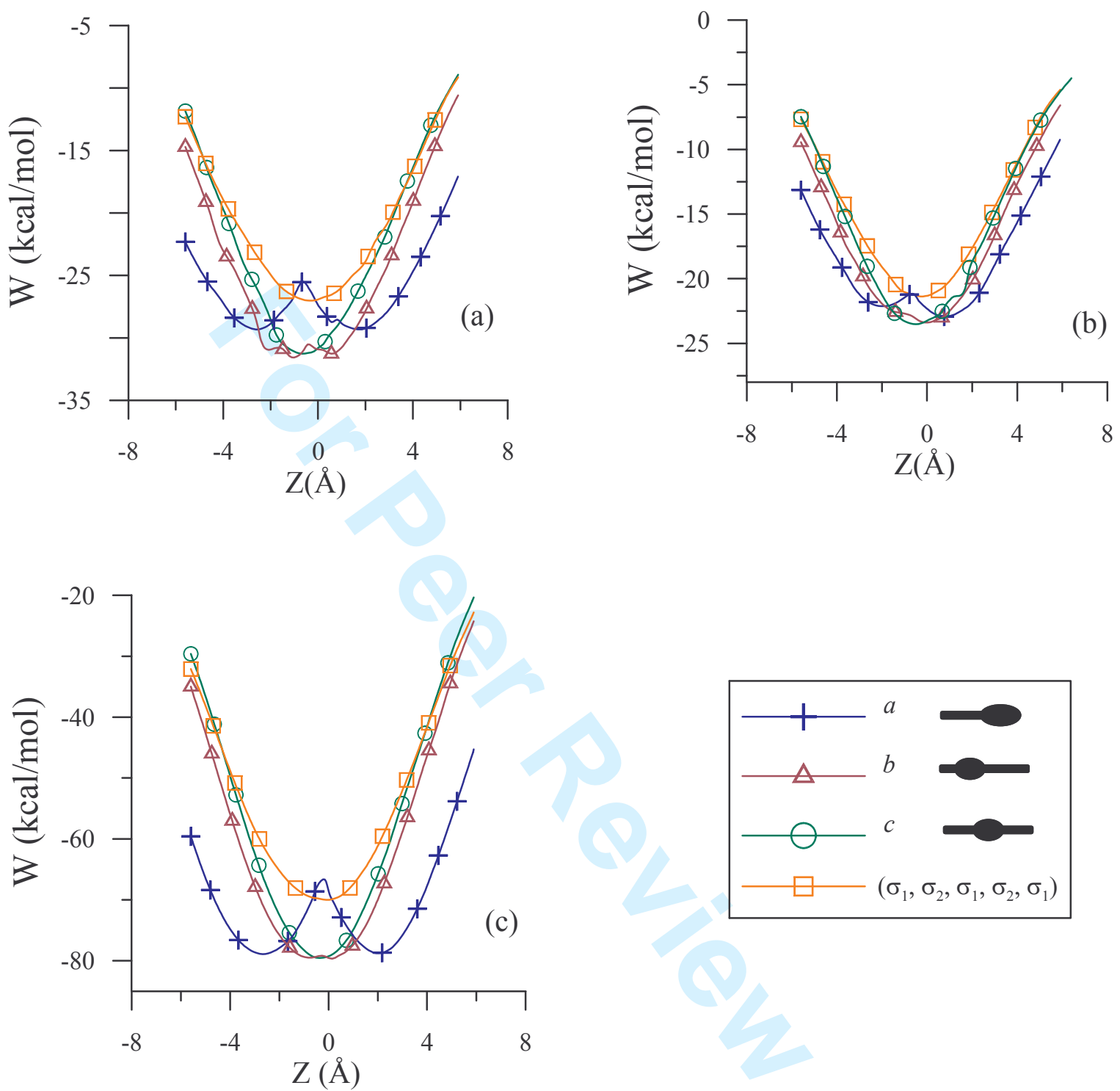

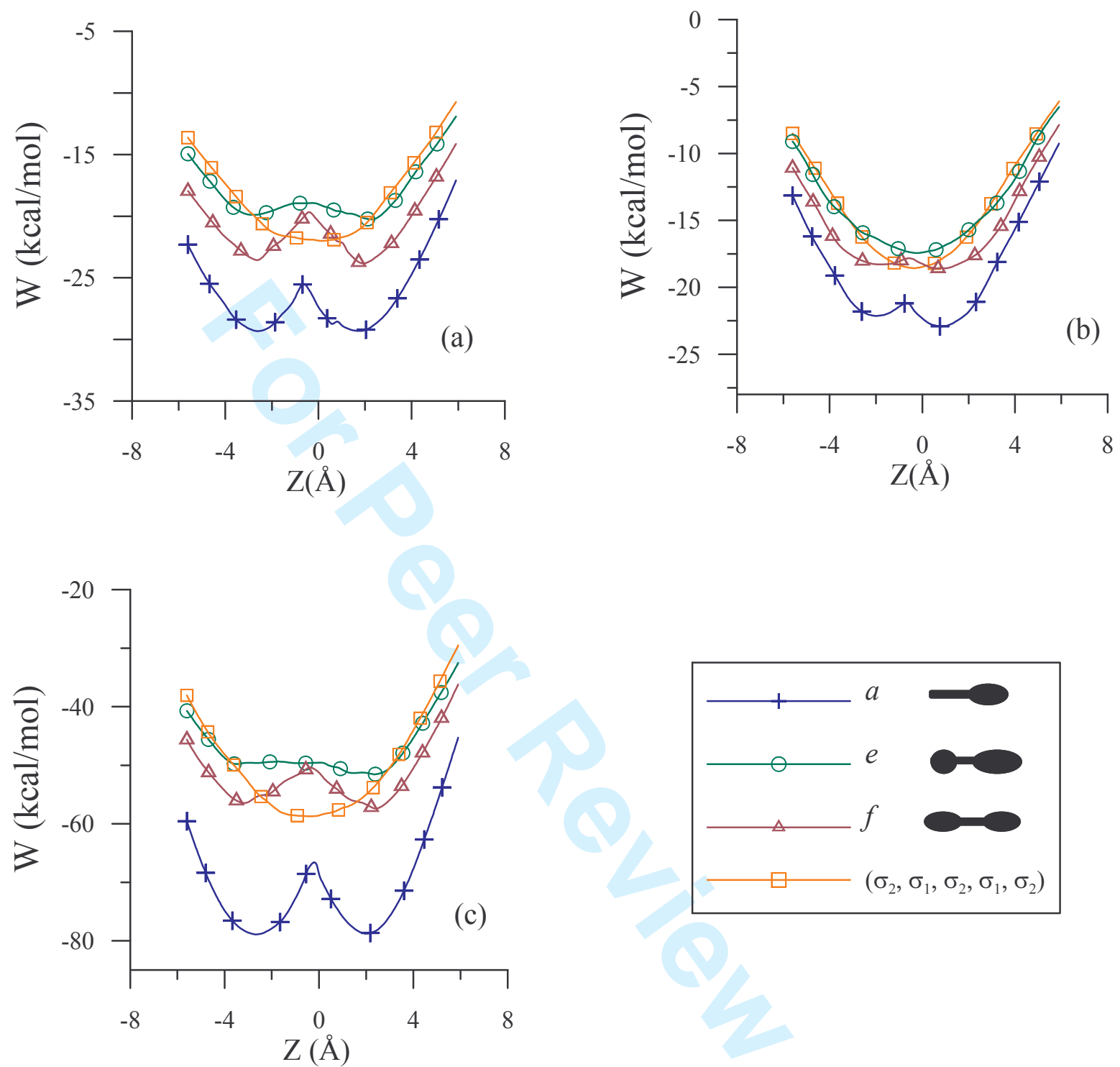

Figure 4 

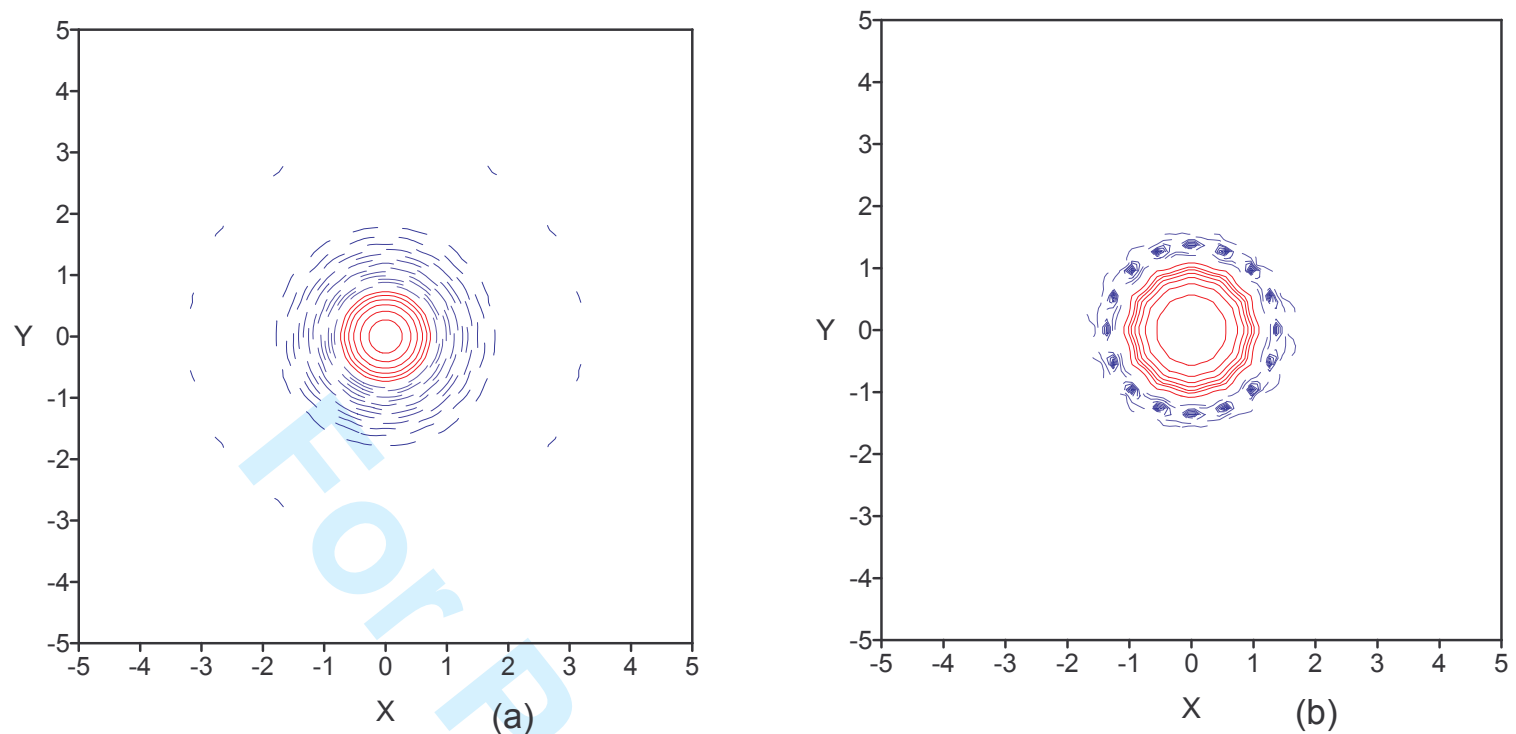

1
2
3
4
5
6
7
8
9
10
11
12
13
14
15
16
17
18
19
20
21
22
23
24
25
26
27
28
29
30
31
32
33
34
35
36
37
38
39
40
41
42
43
44
45
46
47
48
49
50
51
52
53
54
55
56
57
58
59
60
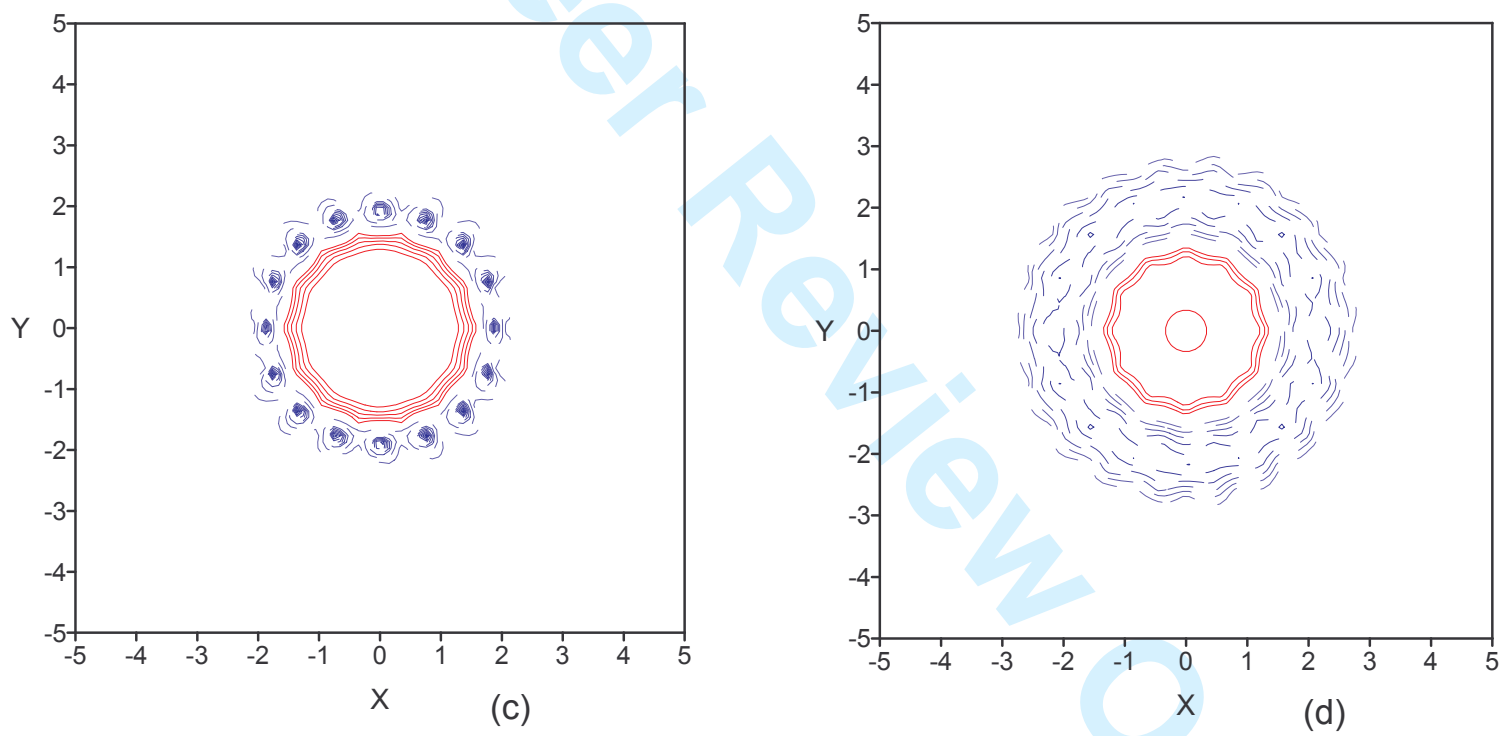

Figure 5

URL: http:/mc.manuscriptcentral.com/tandf/gsch Email: suprachem@mail.cm.utexas.edu 